\title{
Chitin oligosaccharides (oligomer of GlcNAc) protect mycelial growth of Candida albicans in vitro and in vivo.
}

\author{
Sanae A. Ishijima1 ${ }^{1}$, Tamo Fukamizo ${ }^{2}$, Kimihiko Satoh ${ }^{3}$, Takako Noguchi ${ }^{3}$, Yu Guo ${ }^{1}$, TsuyoshiYamada ${ }^{1}$ and Shigeru Abe ${ }^{1}$ \\ ${ }^{1}$ Teikyo University Institute of Medical Mycology, ${ }^{2}$ Kindai University, ${ }^{3}$ Koyo Chemical Co., LTD
}

\begin{abstract}
The amino sugar N-acetylglucosamine (GIcNAc) is well known to induce the mycelial growth of Candida albicans. We had reported that GlcNAc aggravated murine oral and gastrointestinal candidiasis. Here we investigated the effect of the oligomer of GlcNAc on mycelial growth of $C$. albicans and murine oral candidiasis. Surprisingly, chitin oligosaccharides inhibited mycelial growth of $C$. albicans in vitro. Inhibition of mycelial growth detected by the length of hyphae from $4 \mathrm{~h}$-culture of $C$. albicans; hyphae length elongation of $C$. albicans cells in the control culture, 25mM GlcNAc-, (GlcNAc) ${ }_{3}$ - and (GIcNAc) $_{4}$-supplemented culture were 89.77 $\pm 20.38,86.1 \pm 22.7,67.18 \pm 27.96$, and $42.25 \pm 22.4 \mu \mathrm{m}$, respectively. Average length of the elongated hyphae in the case of $(\mathrm{GlcNAc})_{3}$ or $(\mathrm{GlcNAc})_{4}$ were significantly less than control or monomer $(\mathrm{P}<0.01)$. After $20 \mathrm{~h}$ culture, each volumes of hyphae attached to the bottom of the culture plates were measured by crystal violet staining method. The hyphal volume of $C$. albicans after $20 \mathrm{~h}$-culture were decreased by the presence of the oligomer of GlcNAc (degree of polymerization $=2$ to 6 ). The inhibitory effect of mycelial growth were observed in the case of three strains of $C$. albicans, TIMM1768, TIMM2640 and TIMM3163 (having fluconazole-resistant phenotype). To clarify the effect of chitin oligosaccharides in vivo, their effects on experimental murine oral candidiasis were examined. The mice infected with $C$. albicans were administrated 50 $\mu \mathrm{L}$ of $12.5 \mathrm{mM}(\mathrm{GlcNAc}) 1-4$ four times (5min, 3h, 24h, 27h). Although mice given GlcNAc showed significant aggravation of oral symptoms of candidiasis after two days (P<0.01), mice given (GIcNAc) $)_{2-4}$ showed significant improvement when compared with those of GlcNAc in oral symptoms $(\mathbf{P}<0.01)$. We discuss that application of chitin oligosaccharide as a nutritional supplement may have a valuable effect on oral health in people susceptible to oral or gastrointestinal candidiasis.
\end{abstract}

Objectives: The amino sugar N-acetylglucosamine (GlcNAc) is well known to induce the mycelial growth of $C$. albicans. We had reported that GlcNAc aggravated murine oral and gastrointestinal candidiasis ${ }^{1,2)}$, and $C$. albicans adheres to chitin, polymer of GlcNAc ${ }^{3}$. Here we investigated the effect of chitin oligosaccharides, the oligomer of GlcNAc, on mycelial growth of $C$. albicans and murine oral candidiasis.

Methods: C. albicans TIMM1768, TIMM2640 and TIMM3136 from patients with candidiasis were used. Chitin oligosaccharides (GlcNAc) $n$ $(n=1-6)$ were produced by partial hydrolysis of crab chitin by the method of Rupley ${ }^{4}$. One hundred microliters of $C$. albicans cell suspension were aliquoted into 96-well microtiter plates $\left(1 \times 10^{4} \mathrm{cfu} /\right.$ well for germ-tube formation, $1 \times 10^{3} \mathrm{cfu} /$ well for mycelial growth analysis), and $100-\mu 1$ serial dilutions of chitin oligosaccharides (GlcNAc, $(\mathrm{GlcNAc}) 3$ or $(\mathrm{GlcNAc}) 4)$ were added the wells which made final concentration of $50 \mathrm{mM} / \mathrm{ml}$ to 3.13 $\mathrm{mM} / \mathrm{ml}$ and incubated at $37 \mathrm{C}$ in $5 \% \mathrm{CO}_{2}$ in air for $2 \mathrm{~h}$ or $4 \mathrm{~h}$ for germ-tube formation, for $20 \mathrm{~h}$ for mycelial growth analysis. Animal experiments were performed according to the guidelines for the care and use of animals approved by Teikyo University (No.15-026). The experimental procedures for candidiasis model as shown below were according to Takakura ${ }^{5)}$

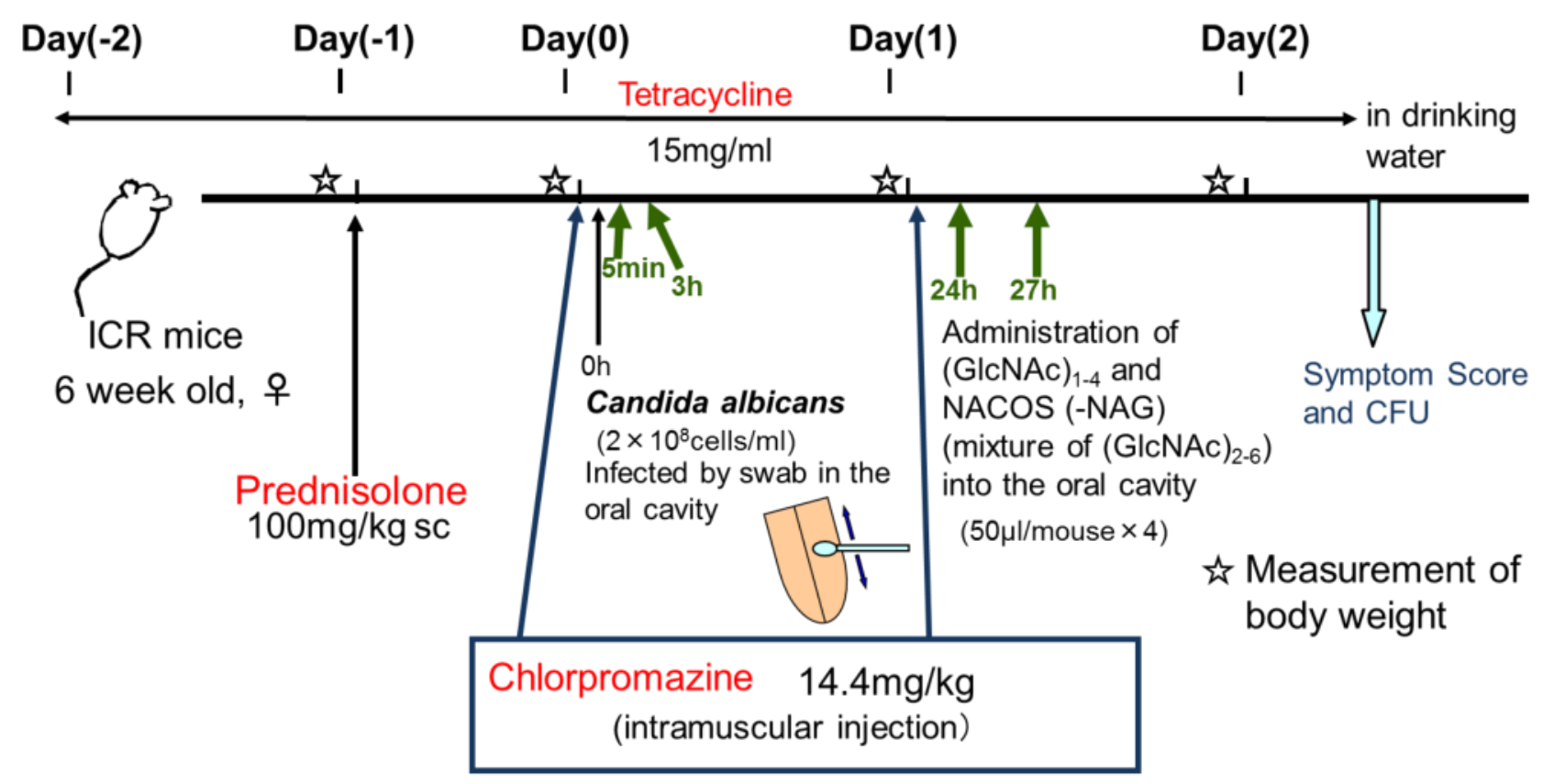

Group of immunosuppressed mice were inoculated C. albicans TIMM1768 cells and $50 \mu \mathrm{L}$ of test samples were placed on the surface of tongues at $5 \mathrm{~min}, 3 \mathrm{~h}, 24 \mathrm{~h}$ and $27 \mathrm{~h}$ after inoculation (Control, $\mathrm{n}=6 ; 25 \mathrm{mM}$ GlcNAc, $\mathrm{n}=6 ; 25 \mathrm{mM}(\mathrm{GlcNAc})_{2}, \mathrm{n}=6 ; 25 \mathrm{mM}(\mathrm{GlcNAc})_{3}, \mathrm{n}=6 ; 25 \mathrm{mM}$ $(\mathrm{GlcNAc})_{4}, \mathrm{n}=6 ; 25 \mathrm{mM}$ NACOS (-NAG) (mixture of $\left.(\mathrm{GlcNAc})_{2-6}\right), \mathrm{n}=6$ ).

\section{Results:}

1. Chitin oligosaccharides inhibited mycelial growth of C. albicans in vitro. Typical images of $C$. albicans TIMM1768 cultured with chitin oligo. A; 2h, B; 4h. a; control, b; 25mM GlcNAc, c; 25mM (GlcNAc) $)_{3}$,

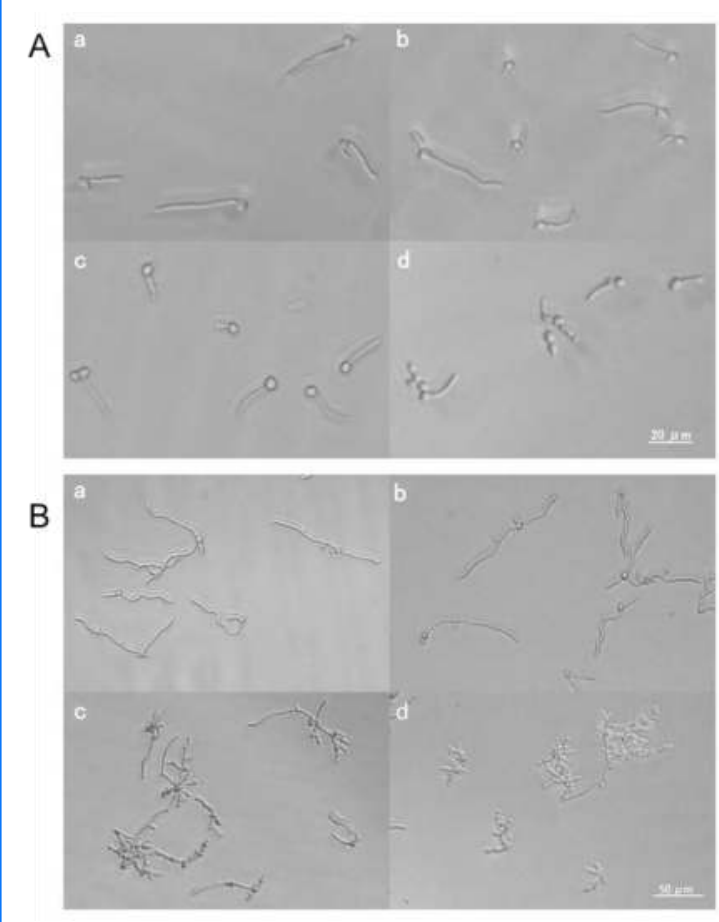
and $\mathrm{d} ; 25 \mathrm{mM}(\mathrm{GlcNAc})_{4}$.

Length of hyphae were measured using Lenaraf 220b software (Vector Japan)

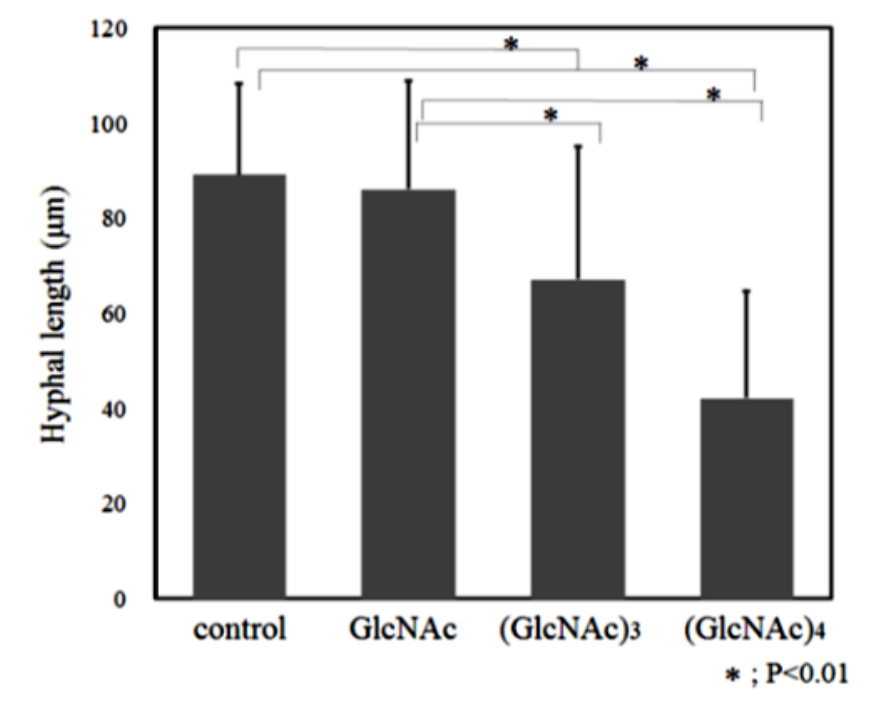

Inhibition of mycelial growth detected by the length of hyphae from $4 \mathrm{~h}$ culture of $\mathrm{C}$. albicans; hyphae length elongation of $\mathrm{C}$. albicans cells in the control culture, $25 \mathrm{mM}$ GlcNAc-, $25 \mathrm{mM}$ (GlcNAc)3- and 25mM (GlcNAc)4supplemented culture were $89.77 \pm 20.38,86.1 \pm 22.7,67.18 \pm 27.96$, and $42.25 \pm 22.4 \mu \mathrm{m}$, respectively. Average length of the elongated hyphae in the case of (GlcNAc) 3 or (GlcNAc)4 were significantly less than control or monomer $(\mathrm{P}<0.01)$.

2. The hyphal volume of $C$. albicans after $20 \mathrm{~h}$-culture were decreased by the presence of the oligomer of GlcNAc (degree of polymerization $=2$ to 6 ).

After 20h culture, each volumes of hyphae attached to the bottom of the culture plates were measured by crystal violet method.

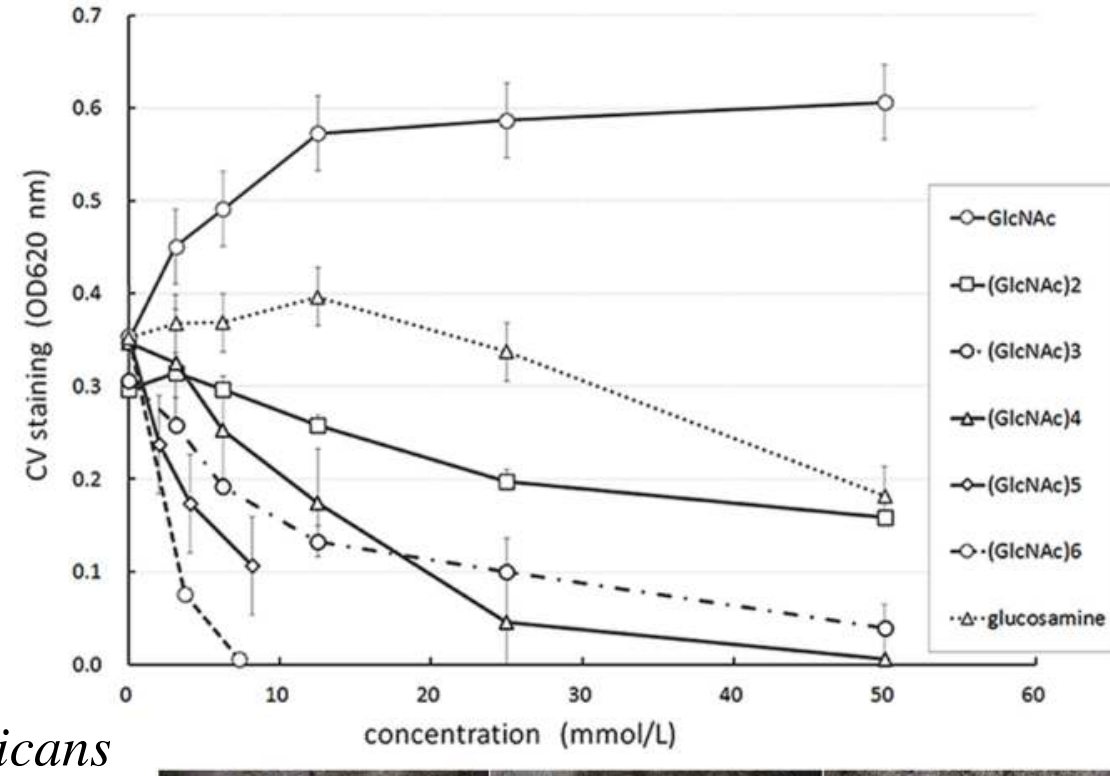

Typical images of C. albicans cultured with $(\mathrm{GlcNAc})_{1-6}$ were shown in the right figure.

The mycelial growth inhibition were observed in the case of 3 strains of $C$. albicans, TIMM1768, TIMM2640 and TIMM3163 (FCZ-resistant).

\section{GlcNAc induced symptom} severity of oral candidiasis,

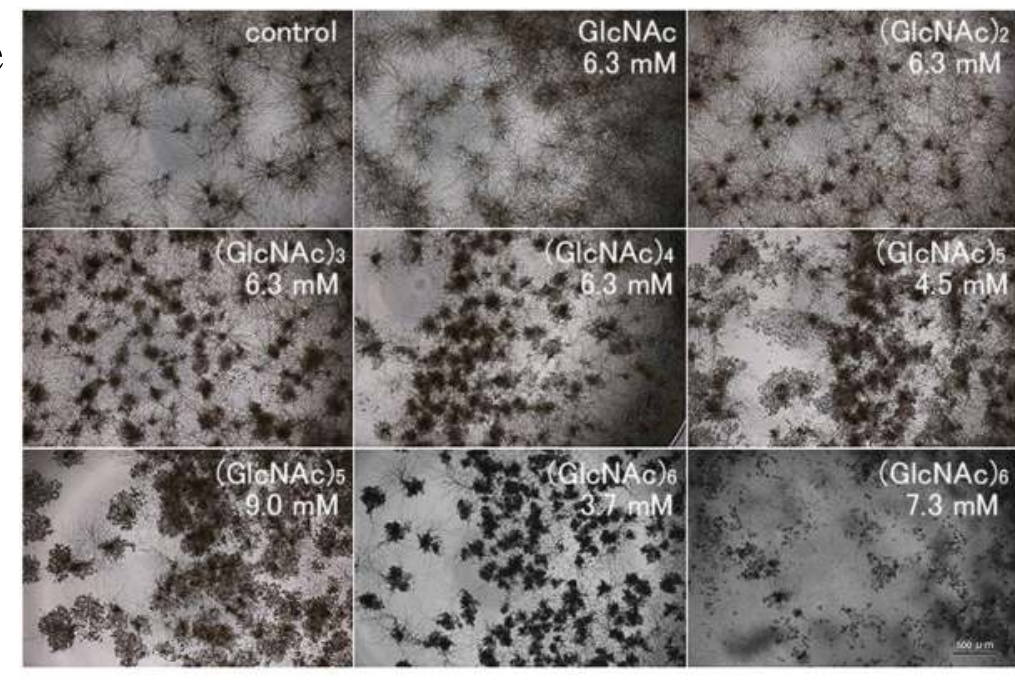
although oligomer of GlcNAc inhibited candidiasis.

The macroscopic evaluation of infection was expressed by scoring lesions from 0 to 4 on the basis of the extent and severity of whitish, curd- like patches (arrows) on the tongue surface according to Takakura ${ }^{5}$ as follows: 0 , normal; 1 , white patches in less than 20\%; 2 , white patches in less than $90 \%$ but more than $91 \%$; 4 , thick white patches like pseudomembranes in more than $91 \%$.

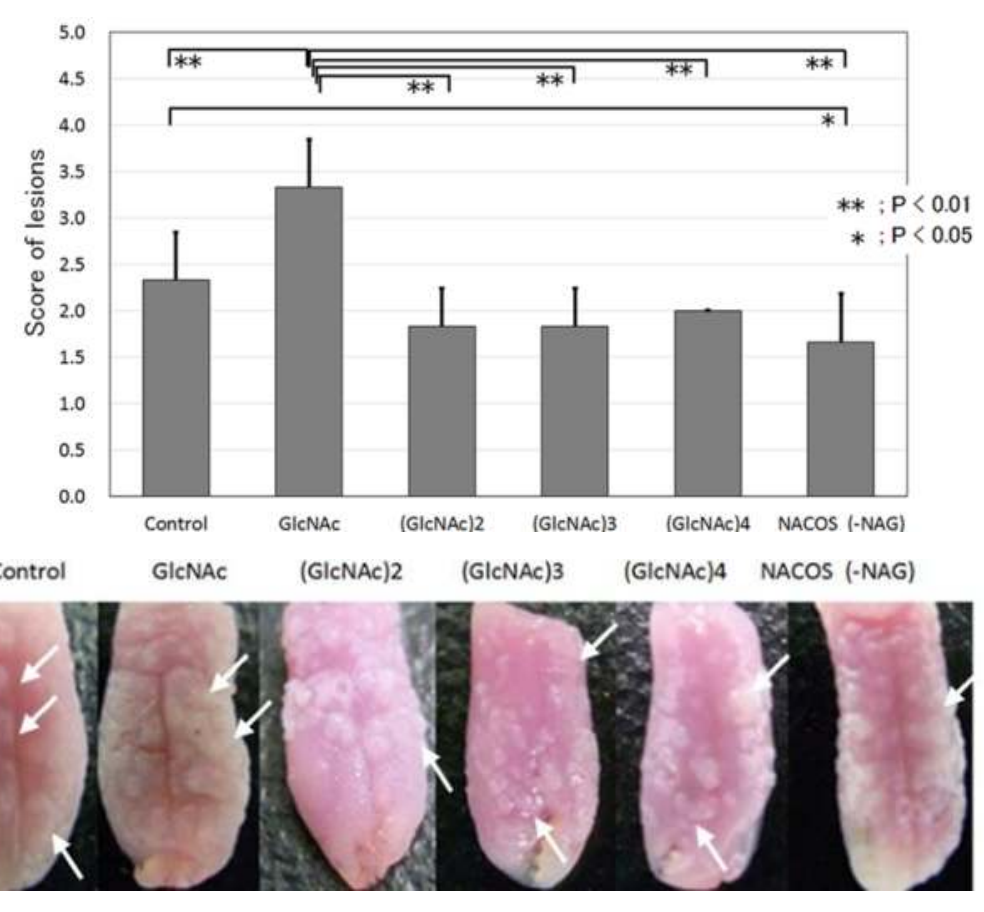

Conclusion: Application of chitin oligosaccharide as a nutritional supplement may have a better effect on oral health in people susceptible to oral or gastrointestinal candidiasis.

Acknowledgements; This work was supported in part by a Grant-in-Aid for scientific research (C) (JP16K08790) to SAI.

Reference; 1. Ishijima SA et al. Med Mycol, 50; 252-258 (2012); 2. Ishijima SA et al. Med Mycol, 56E; E31-E39 (2015); 3. Ishijima SA et al. Med Mycol J, 58E;E15-E21 (2017); 4. Rupley JA. Biochem Biophys Acta, 83; 245-255 (1964); 5. Takakura N et al. Microbiol Immunol 47: 321-326 (2003) 